
\title{
25 Research Soure \\ Consideration of the Optimal Surgical Procedure Based on the Risk of Recurrence in Clinical Stage 0 or IA Lung Adenocarcinoma
}

Tomoyoshi Takenaka ( $\nabla$ ttake@surg2.med.kyushu-u.ac.jp )

National Hospital Organization, Kyushu Medical Center https://orcid.org/0000-0002-0278-1854

Tetsuzo Tagawa

Kyushu University

Naoki Hartake

Kyushu University

Fumihiko Kinoshita

Kyushu University

Yuki Ono

Kyushu University

Sho Wakasu

Kyushu University

Yuka Oku

Kyushu University

Masaki Mori

Kyushu University: Kyushu Daigaku

\section{Research article}

Keywords: lung adenocarcinoma, TNM staging, surgical resection, recurrence

Posted Date: March 19th, 2021

DOl: https://doi.org/10.21203/rs.3.rs-330128/v1

License: (c) (i) This work is licensed under a Creative Commons Attribution 4.0 International License.

Read Full License

Version of Record: A version of this preprint was published at Anticancer Research on January 29th, 2022. See the published version at https://doi.org/10.21873/anticanres.15577. 


\section{Abstract \\ Background}

Although sublobar resection is widely performed for early-stage non-small cell lung cancer, what kind of patients should be actively indicated for sublobar resection has not yet been established according to 8th edition of tumor node metastasis classification (TNM). We evaluated the computed tomography (CT) features and optimal surgical procedures of clinical stage 0 or IA adenocarcinoma from the perspective of recurrence.

\section{Methods}

We retrospectively investigated 508 lung adenocarcinoma diagnosed as c-stage 0 or IA in the 8th edition of TNM classification. A survival analysis was performed according to the clinical T descriptor, CT features and surgical procedures.

\section{Results}

The tumors were classified as follows: 74 with pure ground glass opacity (GGO), 237 part-solid tumors and 197 solid tumors. The types of surgical procedures were lobectomy $(n=328)$, segmentectomy $(n=$ 73) and wedge resection $(n=107)$. Clinical T descriptors were cTis in 74 patients, cT1 mi in 68 patients, cT1a in 94 patients, cT1b in 181 patients and cT1c in 91 patients. Recurrence was observed 46 cases (9\%), including 3 (3.1\%) with cT1a, 23 (12.7\%) with cT1b and 20 (22.0\%) with cT1c. No recurrence was observed in cTis or cT1 mi cases. Solid tumors with cT1b recurred more often than part-solid tumors among cT1b cases $(6.8 \%$ vs. $16.8 \%)(p=0.046)$. There were no marked differences in the recurrence rate between part-solid and solid tumors in the cT1a and cT1c groups. The patients who received sublobar resection developed recurrence more often than the patients who received lobectomy among cT1 $\mathrm{b}$ cases $(10.1 \%$ vs. $21.4 \%)$ and cT1c cases $(18.0 \%$ vs. $46.2 \%)(p=0.053$ and $p=0.023)$.

\section{Conclusions}

Pure GGO and cT1mi cases should be actively considered for sublobar resection, while cT1b (especially solid cT1b cases) and cT1c cases should be considered for lobectomy to prevent recurrence.

\section{Background}

There were 2.0 million incident cases of lung cancer and 1.7 million deaths in 2016; lung cancer continues to be the most common type of cancer [1]. However, widespread screening with low-dose helical computed tomography (CT) has led to an improved rate of early detection for small-sized lung cancers [2]. 
Since the 1970s, tumor size has been a primary descriptor, and a significant prognostic factor for lung cancer in the tumor, node, metastasis (TNM) classification of the staging system of the Union for International Cancer Control [3]. In the 8th edition of the TNM classification for non-small cell lung cancer (NSCLC), the cT category is defined by the tumor size, but only the size of the solid component on CT for prognostic stratification [4]. Recently, several researchers have reported that radiologically determined pure-solid lung cancers without a ground glass opacity (GGO) component exhibit more malignant behavior than the tumors with GGO [5-7]. Thus, whether or not patients with solid tumors should be treated similarly to patients with part-solid tumors with equal-sized solid components is controversial.

Although lobectomy is the standard resection approach for patients with clinical stage I NSCLC who can tolerate lobectomy, according to a previous randomized controlled trial comparing lobectomy with sublobar resection including segmentectomy and wide-wedge resection [8], sublobar resection is widely performed for early-stage NSCLC due to its improved diagnostic ability for cases with a good prognosis $[9,10]$. According to The Japanese Joint Committee of Lung Cancer Registry reports, $22.7 \%$ of cases of lung cancer resection cases received sublobar resection in 2010 [10]. In addition, a phase III study was conducted to evaluate the non-inferiority of segmentectomy compared with lobectomy in patients with small peripheral NSCLC ( $\leq 2 \mathrm{~cm}$ diameter) in Japan and in the United States [11, 12]. However, what kind of patients should be actively indicated for sublobar resection has not yet been established.

In the present study, we assessed the CT features and optimal surgical procedures for clinical stage 0 or IA adenocarcinoma according to the current staging system from the perspective of recurrence.

\section{Methods}

\section{Patients}

We included 508 consecutive patients who were diagnosed with clinical stage 0 or IA NSCLC according to the TNM 8th edition, and underwent surgery between January 2009 and December 2018 at our department.

Written informed consent was obtained from each patient to use their medical records. This study was approved by the institutional review of board of Kyushu University Hospital (IRB number: 2019 - 232).

\section{The perioperative examination}

Contrast-enhanced CT was routinely performed during each preoperative examination of the entire lung except for contraindicated cases. Images were viewed on standard lung windows (level: -500 to -700 $\mathrm{HU}$; width: 1500 to $2000 \mathrm{HU}$ ). The whole tumor size and solid component size were determined from the maximum diameter of the tumors and the solid part of the tumors, respectively. Tumors were divided into pure GGO, part-solid tumors and solid tumors based on the consolidation tumor ratio (CTR) in thin-section CT: pure GGO, CTR = 0; part-solid, $0<C T R<1$; solid, $C T R=1$. Clinical staging was determined based on the findings of chest and upper abdomen $\mathrm{CT}$, brain $\mathrm{CT}$ or magnetic resonance imaging (MRI), radionuclide 
bone scans and/or with fluorodeoxyglucose-positron emission tomography (FDG-PET). Endobronchial ultrasound mediastinal lymph node biopsies were performed when nodal involvement was suspected. The histologic types of the lung adenocarcinoma were classified according to the International Association for the Study of Lung Cancer /American Thoracic Society/European Society of Thoracic Surgeons classification [13].

Follow-up examinations were usually conducted every three to four months for the first two years and every three to six months thereafter. Routine follow-up procedures included a physical examination, hematological examination and chest radiography. In addition, chest and abdominal CT were performed at least once a year. If recurrent disease was suspected, further evaluations, such as MRI and FDG-PET were added. Recurrent NSCLC was diagnosed based on physical examinations and diagnostic imaging findings consistent with recurrent disease. When clinically feasible, diagnoses were histologically confirmed.

The date of recurrence was defined when recurrence was histologically proven or, in cases that were diagnosed by clinical evidence, when recurrent disease was recognized by the attending physician.

\section{Operation Policy}

With regard to the agreement at our institution, if a tumor was pure GGO or GGO-dominant, the patients actively received sublobar resection, whereas major lung dissection with systematic or selective lymph node dissection was warranted for part-solid or solid tumors. Some patients who were elderly, had a high cardiopulmonary risk or could not tolerate lobectomy for any other reason reluctantly received sublobar resection.

\section{Data collection and extraction}

Demographic, clinical and treatment data were extracted from an institutional database that included all patients who had undergone thoracic surgery. For cases before 2017, clinical T staging was reassigned in accordance with the 8th edition of the TNM classification.

\section{Statistical analyses}

Comparisons of continuous and dichotomous variables between groups were performed with Student's $t$ test and the $\chi^{2}$-test, respectively. The probability of a survival was estimated by the Kaplan-Meier method. Differences in the survival were evaluated by log-rank tests. The analyses were performed using the JMP software package (version 11, SAS Institute Inc., Cary, North Carolina). $P<0.05$ was considered significant.

\section{Results}

The characteristics of the 508 patients are summarized in Table 1. Preoperative chest CT showed 74 pure GGO cases, 237 part-solid tumors and 197 solid tumors. The types of procedures included lobectomy (n $=328)$, segmentectomy $(n=73)$ and wedge resection $(n=107)$. The median duration of follow-up after 
surgical resection was 49 months (range: 1-121 months). The mean tumor measurements were a whole tumor size of $18 \mathrm{~mm}$ (range: $5-30 \mathrm{~mm}$ ) and solid component size of $12 \mathrm{~mm}(0-30 \mathrm{~mm})$ (Table 1). The clinical T descriptor according to the TNM 8th edition was cTis in 74 patients (15\%), cT1 mi in 68 patients (13\%), cT1a in 94 patients (19\%), cT1b in 181 patients (36\%), and cT1c in 91 patients (18\%) (Table 1). 
Table 1

Patients' characteristics

\begin{tabular}{|c|c|}
\hline Variable & $\mathrm{n}=\mathbf{5 0 8}$ \\
\hline Age (range) (years) & 67 (33 to 88) \\
\hline \multicolumn{2}{|l|}{ Gender } \\
\hline Male & $239(47 \%)$ \\
\hline Female & $269(53 \%)$ \\
\hline \multicolumn{2}{|l|}{ CT features } \\
\hline Pure GGO & $74(15 \%)$ \\
\hline Part-solid & $237(47 \%)$ \\
\hline Solid & $197(39 \%)$ \\
\hline Whole tumor size (mean) & $5-30 \mathrm{~mm}(18 \mathrm{~mm})$ \\
\hline Solid component size (mean) & $0-30 \mathrm{~mm}(12 \mathrm{~mm})$ \\
\hline \multicolumn{2}{|c|}{ cT descriptor according to TNM 8th edition } \\
\hline Tis & $74(15 \%)$ \\
\hline T1mi & $68(13 \%)$ \\
\hline T1a & $94(19 \%)$ \\
\hline $\mathrm{T} 1 \mathrm{~b}$ & $181(36 \%)$ \\
\hline $\mathrm{T} 1 \mathrm{c}$ & $91(18 \%)$ \\
\hline \multicolumn{2}{|l|}{ Surgical procedure } \\
\hline Lobectomy & $328(65 \%)$ \\
\hline Segmentectomy & $73(14 \%)$ \\
\hline Wedge resection & $107(21 \%)$ \\
\hline \multicolumn{2}{|l|}{ pTNM stage 8th edition } \\
\hline Stage 0 & $35(7 \%)$ \\
\hline Stage IA1 & $142(28 \%)$ \\
\hline Stage IA2 & $185(36 \%)$ \\
\hline Stage IA3 & $51(10 \%)$ \\
\hline Stage IB & $50(10 \%)$ \\
\hline
\end{tabular}




\begin{tabular}{|ll|}
\hline Variable & $\mathbf{n = 5 0 8}$ \\
\hline Stage II & $20(4 \%)$ \\
\hline Stage III & $21(4 \%)$ \\
\hline Stage IVa & $4(1 \%)$ \\
\hline CT, computed tomography; GGO, ground glass opacity; TNM, tumor node metastases \\
\hline
\end{tabular}

For the entire cohort, the 5-year overall survival (OS) rate was $93.8 \%$, and the 5 -year disease-free survival (DFS) rate was $87.5 \%$. Kaplan-Meier analyses of the OS and DFS according to the clinical T descriptors on the 8th edition of the TNM classification are shown in Fig. 1A and 1B. The respective 5-year OS and DFS rates by clinical descriptors were as follows: $97.6 \%$ and $97.6 \%$ in cTis, $95.4 \%$ and $95.4 \%$ in cT1 mi, $96.4 \%$ and $93.9 \%$ in $\mathrm{CT} 1 \mathrm{a}, 92.4 \%$ and $84.8 \%$ in $\mathrm{cT} 1 \mathrm{~b}$ and $89.2 \%$ and $70.8 \%$ in $\mathrm{CT} 1 \mathrm{c}$ (Fig. $1 \mathrm{~A}$ and $1 \mathrm{~B}$ ). The correlation between the surgical procedure and CT features or clinical T descriptors is shown in Table 2. The patients with solid tumors or a large number of $T$ descriptors received lobectomy more often than others $(p<0.001$ and $p<0.001)$ (Table 2$)$.

Table 2

Relationship between CT features, clinical T factor and surgical procedure

\begin{tabular}{|llll|}
\hline Variable & Lobectomy & Sublobar resection & \\
\hline & $(\mathrm{n}=328)$ & $(\mathrm{n}=180)$ & $\mathrm{p}$-value \\
\hline CT features & & & \\
Pure GGO & $29(39.2 \%)$ & $45(60.8 \%)$ & $\mathrm{p}<0.001$ \\
\hline Part-solid & $143(60.1 \%)$ & $95(39.9 \%)$ & \\
Solid & $156(79.6 \%)$ & $40(20.4 \%)$ & \\
CT descriptor according to TNM 8th edition & & & $\mathrm{p}<0.001$ \\
\hline Tis & $29(39.2 \%)$ & $45(60.8 \%)$ & \\
\hline T1mi & $31(45.6 \%)$ & $37(54.4 \%)$ & \\
\hline T1a & $52(55.3 \%)$ & $42(44.7 \%)$ & \\
\hline T1b & $139(76.8 \%)$ & $42(23.2 \%)$ & \\
\hline T1c & $77(84.6 \%)$ & $14(15.4 \%)$ & \\
\hline CT, computed tomography; GGO, ground glass opacity; TNM, tumor node metastases & \\
\hline
\end{tabular}

Recurrence was observed in 46 cases (9\%) including 11 patients with part-solid tumors (4.6\%) and 35 patients with solid tumors (17.9\%) (Table 3). Recurrence occurred in 3 patients (3.1\%) with cT1a, 23 patients (12.7\%) with cT1b and 20 patients (22.0\%) with cT1c. No recurrence was observed in pure GGO, 
cTis or cT1 $1 \mathrm{mi}$ cases (Table 3). The presence or absence of recurrence and CT features or surgical procedure in $\mathrm{cT} 1 \mathrm{a}, \mathrm{T} 1 \mathrm{~b}$ and $\mathrm{cT} 1 \mathrm{c}$ cases is shown in Table 4. For $\mathrm{CT} 1 \mathrm{a}$ cases, the CT features and surgical procedure did not influence the recurrence rate; however, for cT1b cases, solid cases developed recurrence significantly more often than part-solid cases $(p=0.046)$. Although it was not statistically significant, patients who received sublobar resection developed recurrence more often than the patients who receive lobectomy $(p=0.053)$. For $\mathrm{cT} 1 \mathrm{c}$ cases, there were no significant difference in the recurrence rate between part-solid cases and solid cases; however, the patients who received sublobar resection developed recurrence more often recurrence than the patients who received lobectomy $(p=0.023)$.

Table 3

Recurrence rate according to CT features and clinical T factor

\begin{tabular}{|llll|}
\hline Variable & Recurrence & Not recurrence & \\
\hline & $(\mathrm{n}=46)$ & $(\mathrm{n}=462)$ & p-value \\
\hline CT features & & & \\
\hline Pure GGO & $0(0 \%)$ & $45(60.8 \%)$ & $\mathrm{p}<0.001$ \\
\hline Part-solid & $11(4.6 \%)$ & $95(39.9 \%)$ & \\
\hline Solid & $35(17.9 \%)$ & $40(20.4 \%)$ & \\
\hline CT descriptor according to TNM 8th edition & & & \\
\hline Tis & $0(0 \%)$ & $74(100 \%)$ & $\mathrm{p}<0.001$ \\
\hline T1mi & $0(0 \%)$ & $68(100 \%)$ & \\
\hline T1a & $3(3.1 \%)$ & $91(96.8 \%)$ & \\
\hline T1b & $23(12.7 \%)$ & $158(87.3 \%)$ & \\
\hline T1c & $20(22.0 \%)$ & $71(78.0 \%)$ & \\
\hline CT, computed tomography; GGO, ground glass opacity; TNM, tumor node metastases & \\
\hline
\end{tabular}


Table 4

Recurrence rate according to tumor morphology and surgical procedures in CT1a, T1b and T1c cases.

\begin{tabular}{|llll|}
\hline cT1a cases & Recurrence & Not recurrence & \\
\hline Variable & $(\mathrm{n}=3)$ & $(\mathrm{n}=91)$ & $\mathrm{p}$-value \\
\hline CT features & & & \\
\hline Part-solid & $2(2.5 \%)$ & $79(97.5 \%)$ & $\mathrm{p}=0.320$ \\
\hline Solid & $1(7.7 \%)$ & $12(92.3 \%)$ & \\
\hline Surgical procedure & & & \\
\hline lobectomy & $3(5.8 \%)$ & $49(94.2 \%)$ & $\mathrm{p}=0.114$ \\
\hline Sublobar resection & $0(0 \%)$ & $42(100 \%)$ & \\
\hline cT1b cases & Recurrence & Recurrence & \\
\hline & $(\mathrm{n}=23)$ & $(\mathrm{n}=158)$ & \\
\hline CT features & & & \\
\hline Part-solid & $5(6.8 \%)$ & $69(93.2 \%)$ & $\mathrm{p}=0.046$ \\
\hline Solid & $18(16.8 \%)$ & $89(83.2 \%)$ & \\
\hline Surgical procedure & & & \\
\hline lobectomy & $14(10.1 \%)$ & $125(89.9 \%)$ & $\mathrm{p}=0.053$ \\
\hline Sublobar resection & $9(21.4 \%)$ & $33(78.5 \%)$ & \\
\hline cT1c cases & Recurrence & Not recurrence & \\
\hline CT features & $(\mathrm{n}=20)$ & $(\mathrm{n}=71)$ & \\
\hline Part-solid & $4(25.0 \%)$ & $12(75.0 \%)$ & \\
\hline Solid & $16(21.3 \%)$ & $59(78.7 \%)$ & \\
\hline Surgical procedure & & & \\
\hline lobectomy & $14(18.0 \%)$ & $64(82.0 \%)$ & \\
\hline Sublobar resection & $6(46.2 \%)$ & $7(53.8 \%)$ & \\
\hline CT, computed tomography & & \\
\hline
\end{tabular}


According to the 8th edition of the TNM classification for NSCLC, the T classifications of the tumors $\leq 3$ in diameters are based only on the size of the solid component on CT or the size of the invasive component at the pathologic examination, as the size of the solid/invasive component is considered to determine the prognosis [4]. In the present study, we first compared OS and DFS of all cases according to the clinical T factor of the TNM 8th edition in clinical stage 0 or IA lung adenocarcinoma. These results indicated that the prognosis of all cases was well stratified according to the classification in this study. However, on comparing the DFS values for CT1 $1 \mathrm{mi}$ and cT1 1 , the 5-year DFS rate was about $25 \%$ different, despite being the same clinical IA stage; therefore, the current treatment strategy, such as the timing of treatment and surgical procedure should be considered carefully. In addition, although this stratification using the classification is useful with regard to the prognosis as well, several issues must be addressed due to switching from the conventional evaluation method involving the maximum tumor diameter. One problems is that the suitability of current treatment strategies, such as limited resection, adjuvant therapy and follow-up, is considered based on the previous staging system.

Although lobectomy is the standard procedure for managing early stage NSCLC [8], sublobar resection, such as segmentectomy and wedge resection, are widely performed for small NSCLC in clinical practice $[9,10]$. Thus, randomized phase III studies such as Cancer and Leukemia Group B (CALBG) 140503 and Japan Clinical Oncology Group (JCOG) 0802 were conducted in United States and Japan to evaluate the non-inferiority of segmentectomy compared with lobectomy in patients with small peripheral NSCLC. The CALBG and JCOG studies targeted NSCLC patients with a whole tumor size of $\leq 2 \mathrm{~cm}[11,12]$. Therefore, the whole tumor size, including ground-glass and lepidic components on radiology and pathology is required in order to evaluate separately from new TNM classification.

It is also necessary to consider surgical procedures according to the current staging system; therefore, we evaluated the optimal surgical procedures for clinical stage 0 and IA adenocarcinoma according to the CT features and current $\mathrm{T}$ descriptors in this study. Our results showed no recurrence in cTis and $\mathrm{cT} 1 \mathrm{mi}$ cases, regardless of surgical procedure; therefore, we may actively consider performing sublobar resection for cTis and CT1 mi cases. In addition, regarding cT1 a cases, only $3 \%$ of all such cases experienced recurrence (all 3 recurrent cases received lobectomy); therefore, the indication of those cases for sublobar resection is appropriate. Regarding cT1b and cT1c cases, patients who received sublobar resection experienced recurrence more often than others. Although the recurrence rate of solid tumors was significantly higher than that of part-solid tumors among cT1b cases, no significant difference was observed between part-solid and solid tumors among cT1a and cT1c cases.

Several studies have recently indicated that tumors with GGO components have a better prognosis than those without GGO component [5-7]. Fu et al. reported that the presence of GGO components was a strong predictor in patients with invasive pathological stage I NSCLC [6]. Hattori et al. also reported that the presence of GGO component had a marked impact on the favorable prognosis of small lung adenocarcinoma [5]. They found that solid tumors with clinical stagelA1, IA2 and IA3 had a significantly worse prognosis than the part-solid tumors of the same clinical stage [5]. Hattori et al. also reported that segmentectomy and a larger tumor size were independent significant clinical factors of loco-regional

Page 10/15 
recurrence in clinical T1aNOM0 (TNM 7th staging) solid NSCLC [14]. There has also been a report comparing the prognosis of solid and part-solid tumors subjected to lobectomy. Takenaka et al. reported a study comparing part-solid and solid tumors among clinical stage IA NSCLC patients who received lobectomy [15]. Although the patients with solid tumors had a significantly worse outcome than those with part-solid tumors in all cases, the propensity-matched analysis detected no significant difference in the survival between the patients with solid and part-solid tumors when matched according to the size of the solid component [15]. These present and previous findings suggest that, for now, the indications for sublobar resection for solid tumors should be carefully determined, even in cases of small adenocarcinoma.

Several limitations associated with the present study warrant mention. First, this study had a retrospective observational design and used a single-institution Japanese database to enroll only patients who had undergone surgical resections. Second, the surgical procedures were selected according to the individual patients and were not randomized. Although the results of Phase III trials are required to establish evidence supporting limited resection for early stage NSCLC, the results of this study appeared to reflect real world data at the moment. Despite these limitations, the present study clarifies that combined evaluation with the clinical T descriptor and CT features reasonably and properly reflects the recurrence rate.

\section{Conclusions}

In conclusion, a combined evaluation of CT features and T descriptor according to TNM 8th edition is useful for deciding on the surgical procedure for clinical 0 and IA adenocarcinoma from the perspective of recurrence. Pure GGO and cT1 mi cases should be actively considered for sublobar resection, while cT1b (especially solid cT1b cases) and cT1c cases should be considered lobectomy to prevent recurrence.

\section{Abbreviations}

CT

computed tomography

TNM

tumor node metastasis classification

GGO

ground glass opacity

NSCLC

non-small cell lung cancer

CTR

consolidation tumor ratio

MRI

magnetic resonance imaging

Page $11 / 15$ 


\section{FDG-PET}

fluorodeoxyglucose-positron emission tomography

OS

overall survival

DFS

disease-free survival

CALBG

Cancer and Leukemia Group B

JCOG

Japan Clinical Oncology Group

\section{Declarations}

\section{Ethics approval and consent to participate}

Written informed consent was obtained from each patient to use their medical records. This study was approved by the institutional review of board of Kyushu University Hospital (IRB number: 2019-232).

\section{Consent for publication}

Not applicable

\section{Availability of data and materials}

The datasets used and/or analysed during the current study are available from the corresponding author on reasonable request.

\section{Competing interests}

The authors declare that they have no competing interests

\section{Funding}

No funding was received for this work.

\section{Authors' contributions}

(I) Conception and design: Dr. T. Takenaka

(II) Administrative support Drs. T. Tagawa, and M. Mori

(III) Provision of study materials or patients: Drs. T Takenaka and T Tagawa, N Haratake, F Kinoshita

(IV) Collection and assembly of data: Drs. T Takenaka, Y Ono, S Wakasu, Y Oku and F Kinoshita 
(V) Data analysis and interpretation: Dr. T. Takenaka

(VI) Manuscript writing: Dr. T. Takenaka

All authors read and approved final manuscript.

\section{Acknowledgement}

We thank Japan Medical Communications (https://www.japan-mc.co.jp/) for editing drafts of this manuscript.

\section{References}

1. Global Burden of Disease Cancer Collaboration; Fitzmaurice C, Akinyemiju TF, Al Lami FH, Alam T, Alizadeh-Navaei R, Allen C, et al. Global, Regional, and National Cancer Incidence, Mortality, Years of Life Lost, Years Lived With Disability, and Disability-Adjusted Life-Years for 29 Cancer Groups, 1990 to 2016: A Systematic Analysis for the Global Burden of Disease Study. JAMA Oncol. 2018;4:15531568.

2. Aberle DR, DeMello S, Berg CD, Black WC, Brewer B, Church TR, et al. Results of the two incidence screenings in the National Lung Screening Trial. N Engl J Med. 2013;369:920-31.

3. Mountain CF. Revisions in the International System for Staging Lung Cancer. Chest . 1997;111:17101717.

4. Rami-Porta R, Asamura $H$, Travis WD, Rusch VW. Lung cancer-major changes in the American Joint Committee on Cancer eighth edition cancer staging manual. CA Cancer J Clin. 2017;67:138-155.

5. Hattori A, Hirayama S, Matsunaga T, Hayashi T, Takamochi K, Oh S, et al. Distinct Clinicopathologic Characteristics and Prognosis Based on the Presence of Ground Glass Opacity Component in Clinical Stage IA Lung Adenocarcinoma. J Thorac Oncol. 2019;14:265-275.

6. Fu F, Zhang Y, Wen Z, Zheng D, Gao Z, Han H, et al. Distinct Prognostic Factors in Patients with Stage I Non-Small Cell Lung Cancer with Radiologic Part-Solid or Solid Lesions. J Thorac Oncol. 2019;14:2133-2142.

7. Kinoshita F, Toyokawa G, Matsubara T, Kozuma Y, Haratake N, Takamori S, et al. Prognosis of Earlystage Part-solid and Pure-solid Lung. Anticancer Res. 2019;39:2665-2670.

8. Ginsberg RJ, Rubinstein LV. Randomized trial of lobectomy versus limited resection for T1 N0 nonsmall cell lung cancer. Lung Cancer Study Group. Ann Thorac Surg. 1995;60:615-22.

9. Zhang Y, Sun Y, Wang R, Ye T, Zhang Y, Chen H. Meta-analysis of lobectomy, segmentectomy, and wedge resection for stage I non-small cell lung cancer. J Surg Oncol. 2015;111:334-40.

10. Okami J, Shintani Y, Okumura M, Ito H, Ohtsuka T, Toyooka S, et al; Japanese Joint Committee of Lung Cancer Registry. Demographics, Safety and Quality, and Prognostic Information in Both the Seventh and Eighth Editions of the TNM Classification in 18,973 Surgical Cases of the Japanese Joint Committee of Lung Cancer Registry Database in 2010. J Thorac Oncol. 2019;14:212-222. 
11. Nakamura K, Saji H, Nakajima R, Okada M, Asamura H, Shibata T, et al. A phase III randomized trial of lobectomy versus limited resection for small-sized peripheral non-small cell lung cancer (JCOG0802/WJOG4607L). Jpn J Clin Oncol. 2010;40:271-4.

12. Kohman LJ, Gu L, Altorki N, Scalzetti E, Veit LJ, Wallen JM, et al. Biopsy first: lessons learned from cancer and leukemia Group B (CALGB) 140503. J Thorac Cardiovasc Surg. 2017;153:1592-7.

13. Travis WD, Brambilla E, Noguchi M, Nicholson AG, Geisinger KR, Yatabe Y, et al. International Association for the Study of Lung Cancer/American Thoracic Society/European Respiratory Society international multidisciplinary classification of lung adenocarcinoma. J Thorac Oncol. 2011;6:244285.

14. Hattori A, Matsunaga T, Takamochi K, Oh S, Suzuki K. Locoregional recurrence after segmentectomy for clinical-T1aNOMO radiologically solid non-small-cell lung carcinoma. Eur J Cardiothorac Surg. 2017;51:518-525.

15. Takenaka T, Yamazaki K, Miura N, Takeo S. Prognostic ability of new T1 descriptors in the tumour, node and metastasis classification of surgically treated non-small-cell lung cancer Interact Cardiovasc Thorac Surg. 2018;27:714-719

\section{Figures}


Figure $1 \mathrm{~A}$

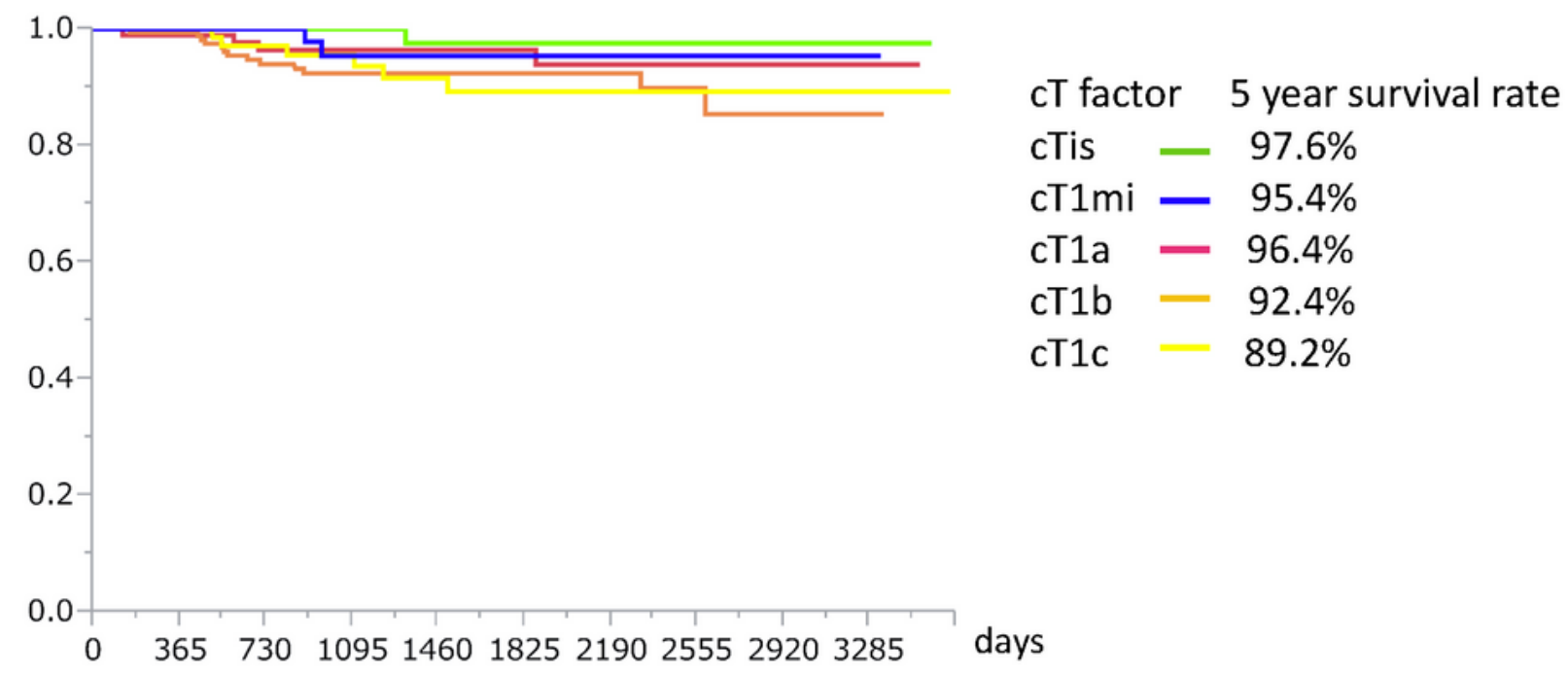

Figure 1B

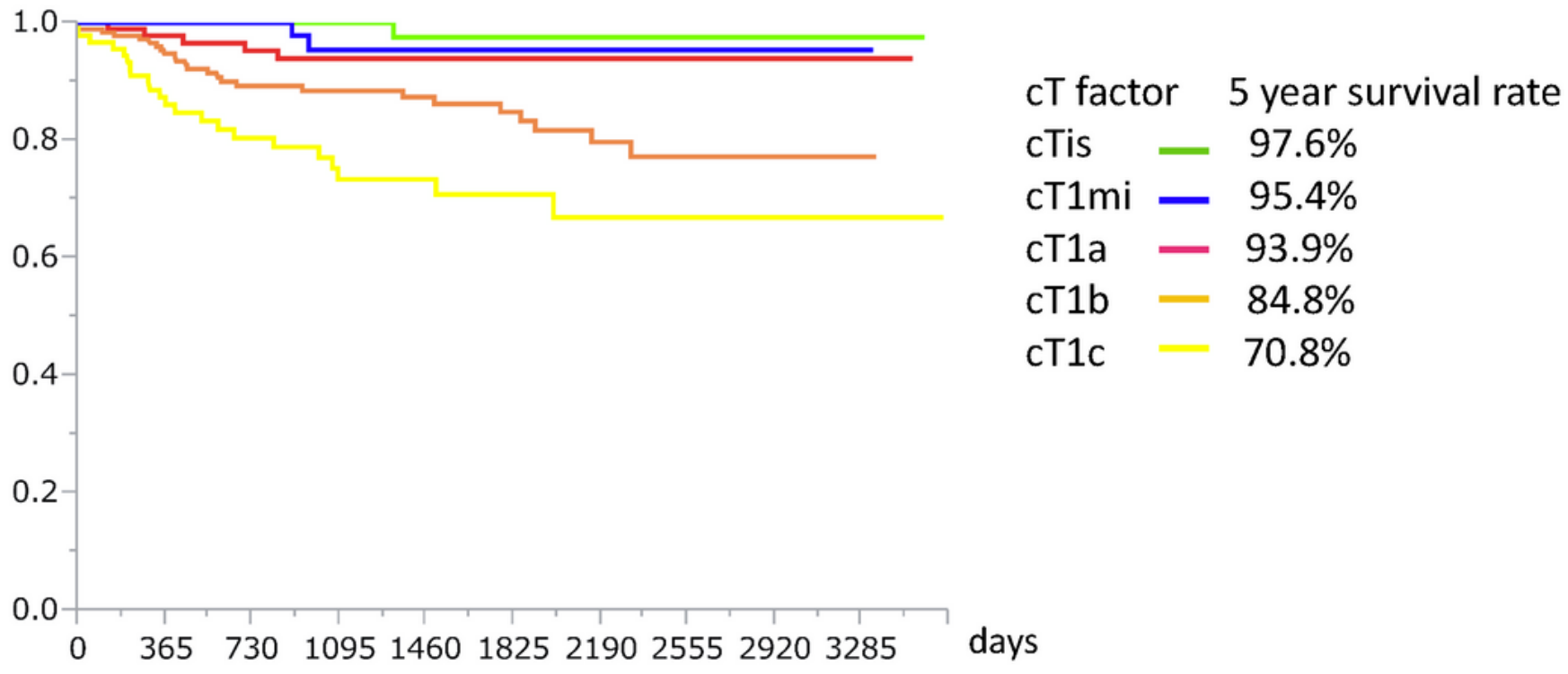

\section{Figure 1}

Kaplan-Meier curves for overall survival (A) and disease-free survival (B) of all cases according to the T descriptors of TNM 8th edition 